\title{
Strong Field Ionization in Arbitrary Laser Polarizations
}

\author{
M. Protopapas, ${ }^{1}$ D. G. Lappas,${ }^{2}$ and P. L. Knight ${ }^{1}$ \\ ${ }^{1}$ Optics Section, Blackett Laboratory, Imperial College London, London SW7 2BZ, United Kingdom \\ ${ }^{2}$ Department of Physics, Lund Institute of Technology, P.O. Box 118, S-221 00 Lund, Sweden
}

(Received 4 June 1997)

\begin{abstract}
We present a new method for investigating the nonperturbative quantum mechanical interaction of light with atoms in two dimensions, without a basis expansion. This enables us to investigate intense laser-atom interactions with light of arbitrary polarization without approximation, within the model restrictions. Results are presented for the dependence of ionization and high harmonic generation on ellipticity seen in recent experiments. Strong evidence of stabilization in circular polarization is found. [S0031-9007(97)04734-0]

PACS numbers: $32.80 . \mathrm{Fb}, 32.80 . \mathrm{Wr}, 42.50 . \mathrm{Hz}$
\end{abstract}

In the past ten years the interaction of intense laser light with atoms has been of great interest because of the many novel nonperturbative phenomena that have been observed, such as above threshold ionization (ATI), high harmonic generation (HHG), and stabilization [1]. The strongly nonperturbative nature of all of these phenomena makes theoretical analysis difficult. However, considerable progress has been made using semianalytic approaches and numerical methods for integrating the Schrödinger equation. The numerical approach has been used extensively in the past ten years, due in part to the increase in available computing power, the lack of approximations (except for those inherent to the Schrödinger equation, the dipole approximation, and the number of spatial dimensions), and the rather limited applicability of analytic approaches. Despite the success of the numerical methods, most investigations have dealt only with linearly polarized light: The reason for this is that at least two dimensions are needed for the case of arbitrary polarization, so established one-dimensional approaches [2] are inappropriate. Current three-dimensional approaches [3-6] are unable to deal with the enormous basis set needed to represent adequately (for arbitrary polarization) the evolving wave function unless a parallel supercomputer [7] is used. The approach used by many authors [3] to describe atomic dynamics in strong laser fields expands the atomic wave function into sums of products of radial wave functions (channels) and angular factors. These channels are coupled together by the very strong laser field, and, for linear polarization, the channel couplings lead to tridiagonal sparse matrices amenable to efficient numerical solution. This approach entirely fails for polarizations other than linear, as the matrix sparseness properties totally disappear [8]. As a result, not much work has been carried out in the past for the general case of atomic response in the plane of an arbitrary laser polarization.

Recent experiments $[9,10]$ on the very strong dependence of high harmonic generation on polarization ellipticity demand theoretical approaches to intense field ionization going beyond the existing methods which rely on linear polarizations. In this Letter, we introduce a unified, fully quantum method for integrating numerically the two-dimensional Schrödinger equation without using a restrictive basis expansion. This enables us to study light of arbitrary polarization and realistic frequencies, interacting within a two-dimensional representation of a hydrogenic atom. New electron dynamics emerge producing unexpected effects in the behavior of strongly distorted atoms. We obtain results showing how the quantum wave packets evolve under the influence of circularly polarized light, contrast them with those obtained using linearly polarized light, and demonstrate the existence of structure within these packets. Fully quantum mechanical results for ionization and high harmonic generation are presented for circular and elliptically polarized light, showing the strong ellipticity dependence seen in recent experiments $[9,10]$. We show that, in the intermediate regime between tunneling and multiphoton ionization, the ionization dependence on ellipticity is opposite to that seen with purely tunneling dynamics; this may be critical for currently planned experiments aimed at generating attosecond pulses.

As in all studies of this type, the starting point is the Schrödinger equation (atomic units are used throughout):

$$
i \frac{\partial}{\partial t} \Psi(x, y, t)=\left[-\frac{1}{2}\left(\frac{\partial^{2}}{\partial x^{2}}+\frac{\partial^{2}}{\partial y^{2}}\right)-\frac{1}{\sqrt{a^{2}+x^{2}+y^{2}}}+(x E \sin \omega t+\epsilon y E \cos \omega t) f(t)\right] \Psi(x, y, t),
$$

where $\Psi(x, y, t)$ is the two-dimensional wave function, $f(t)$ is the pulse envelope, and $\epsilon$ is the ellipticity. The second term on the right-hand side is the smoothed Coulomb potential [2], used to avoid numerical problems associated with the singularity at $x=y=0(a=0.8$ so that the ground state has the same binding energy as hydrogen, i.e., -0.5 a.u.). The pulse envelope, $f(t)$, used here is a two-cycle linear turn-on followed by two cycles 
of constant intensity and then a two-cycle linear turn-off, which ensures no residual drift motion in the free electron displacement. The ellipticity is defined such that $\epsilon=0$ represents linear polarization along the $x$-axis and $\epsilon=1$ circular.

Equation (1) is integrated using a method analogous to that implemented by Grobe and Eberly [11] and others in their studies of one-dimensional two-electron systems in which both electrons are restricted to one dimension. In our case we consider only one electron, but with two degrees of freedom. The core of the approach is the split step method [12] in which the time evolution operator is split into kinetic and potential operators. The action of the kinetic operator is efficiently carried out in Fourier space, while the action of the potential operator is carried out in real space. An absorber is used to remove any part of the wave packet reaching the boundaries, so that artificial reflections are avoided. It is assumed that any wave function reaching the end of the grid box represents ionization, and so the remaining norm gives the necessary information about the amount of ionization occurring. This means that any population in high lying Rydberg states may also be removed; however, this is negligible for the parameter regime of interest here, as we have verified by varying the box dimensions. In all the cases presented, the initial wave function is the ground state, found by imaginary time integration. A more detailed discussion on various computational aspects can be found in Ref. [11].

In Fig. 1 we show the wave packets generated after 3.5 cycles of evolution for the previously described pulse shape with a laser light of wavelength $526 \mathrm{~nm}(\omega=0.0867$ a.u.), i.e., the wavelength of a frequency doubled Nd:YAG laser and intensity $3.51 \times$ $10^{14} \mathrm{~W} \mathrm{~cm}^{-2}$ (0.01 a.u.). For the case of linear polarization it is clear that the wave packet is concentrated about the polarization axis, but there is a non-negligible width due to transverse wave-packet spreading. Interestingly, side lobes can be seen in the wave packet; these are formed by a combination of the slow transverse spreading, the relatively fast laser driven motion in the polarization direction, and the Coulomb attraction (rescattering).

The behavior of the wave packet created by circular polarization is very different. The packet resembles a spiral with modulations within the tail, formed as the wave packet streams away from the atom down the potential barrier in the radial direction. As well as being interesting in their own right, the wave packets show pictorially that the recollisions necessary for efficient HHG can only occur for light with $\epsilon \approx 0$ [9]. The complex structure of the wave function in the linear polarization case reflects the fact that rescattering from the atomic core, according to the recollision model of HHG [13-15], produces strong time-dependent interference patterns in the vicinity of the atomic core, which are responsible for much of the structure in the spectrum
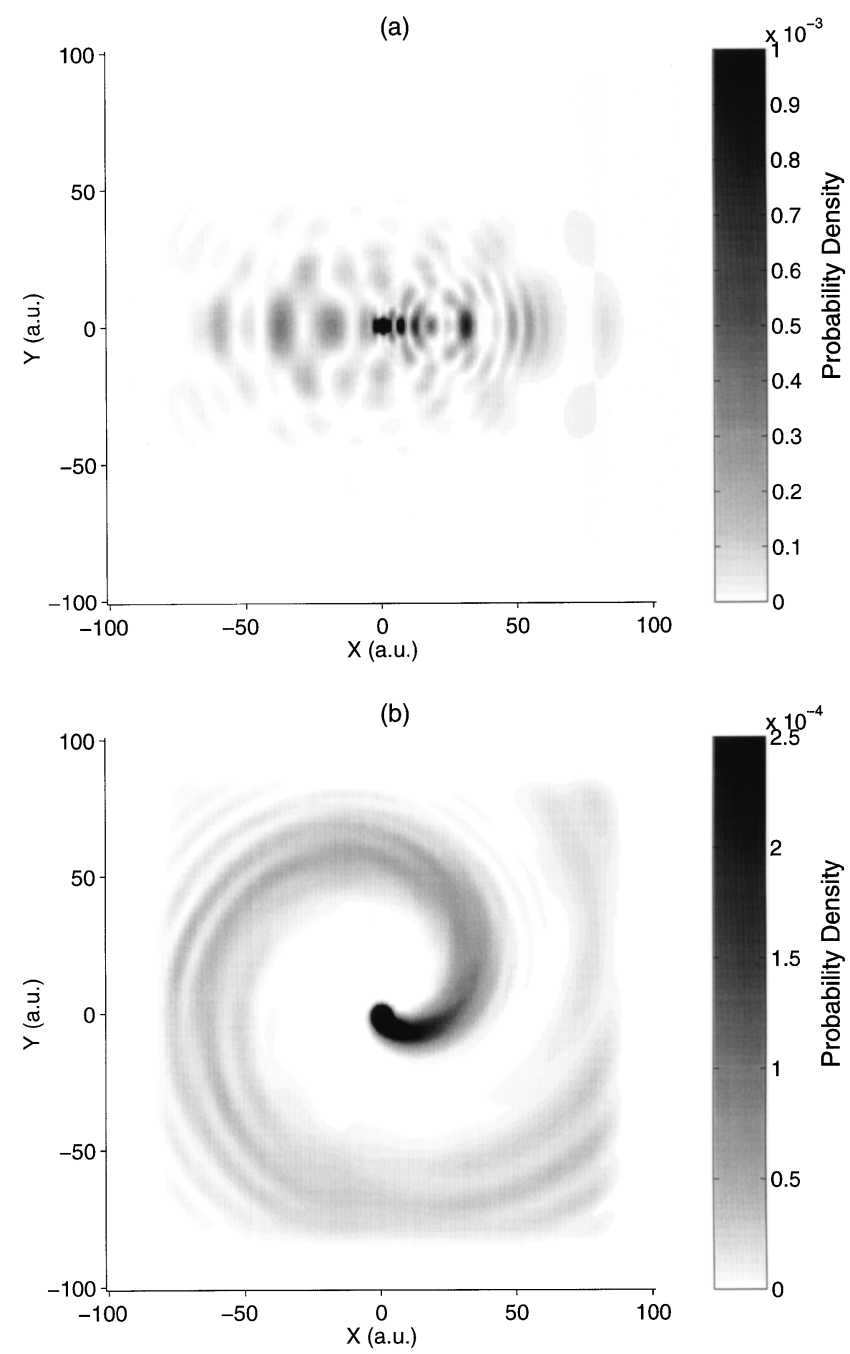

FIG. 1. Snapshots of the probability density taken after 3.5 cycles for (a) linear polarization and (b) circular polarization with intensity $3.51 \times 10^{14} \mathrm{~W} \mathrm{~cm}^{-2}$ and wavelength $526 \mathrm{~nm}$. Distances $(X, Y)$ are shown in atomic units (a.u.).

of HHG [16]. Those interferences are absent in the circular polarization case, as is clear from Fig. 1(b), due to the fact that the probability of rescattering with the atomic core is negligible. We have observed a smooth transition from the recollision behavior, resulting from linear polarization to the swirling tail of the circular polarization, by integrating the wave-packet dynamics for ellipticities ranging from 0 to 1 . This fact can be shown explicitly by investigating the dependence of ionization and HHG spectra on ellipticity. This is shown in Fig. 2(a) where, for a fixed intensity of 0.01 a.u., the normalization at the end of the pulse is plotted as a function of ellipticity [the results have been scaled such that the normalization for the linear case $(\epsilon=0)$ equals unity]. One has to keep in mind that, since the intensity is kept constant for varying ellipticity, the amplitude of the electric field in the initial (linear) polarization direction changes. The reduced ionization in the initial direction is compensated by the possibility of electron ejection in 

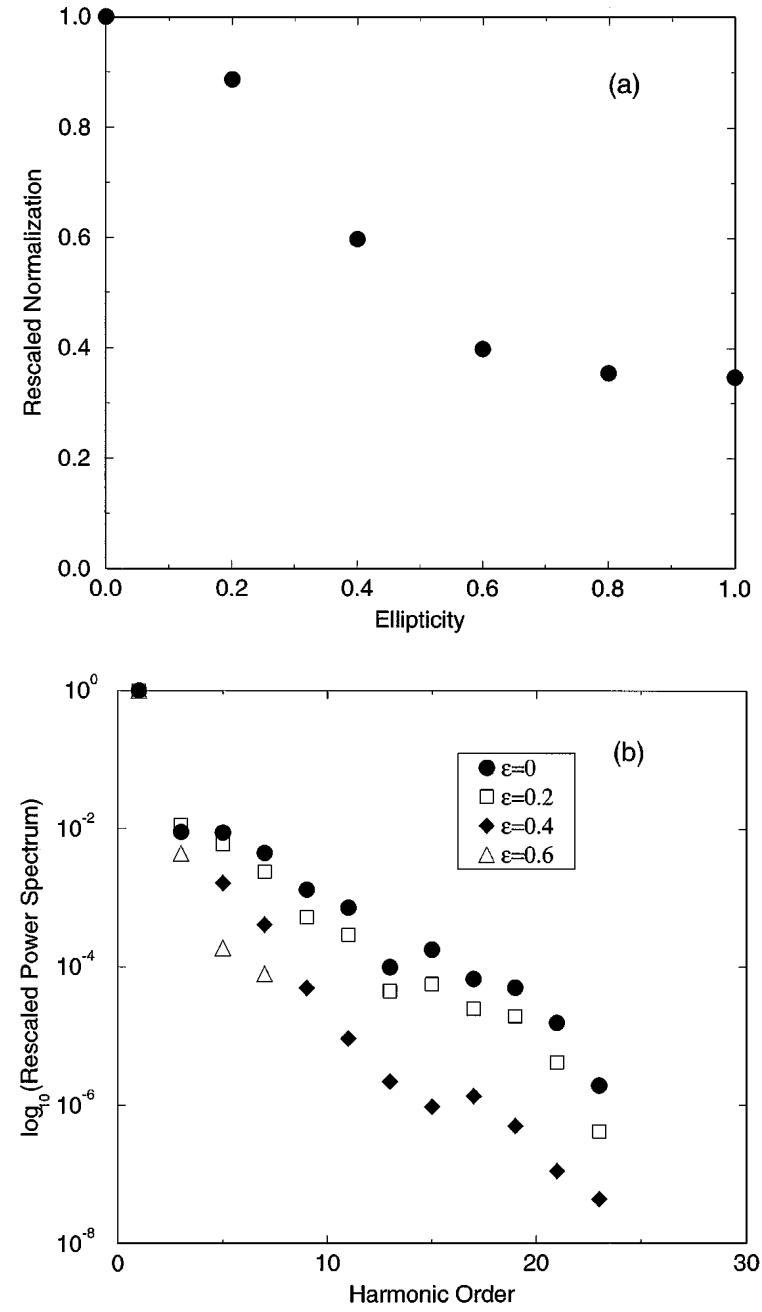

FIG. 2. (a) Rescaled normalization at the end of the pulse as a function of ellipticity for a six-cycle pulse with maximum intensity $3.51 \times 10^{14} \mathrm{~W} \mathrm{~cm}^{-2}$ and wavelength $526 \mathrm{~nm}$. (b) Rescaled HHG spectra as a function of ellipticity for the same laser parameters.

all other directions. The result is a net increase of total ionization yield, especially for such high intensities that over the barrier ionization occurs. This effect is even greater if equal electric field amplitudes are compared rather than equal intensities. In this case the probability of ionization is obviously enhanced, as the electron can escape from the atom by tunneling through or over the barrier at any time in the direction of the instantaneous electric field.

Clearly, for the parameters given above, we find that as the ellipticity is increased the amount of ionization increases by a factor of approximately 3 . Past experiments $[17,18]$ have found that the threshold for ionization is higher for circular polarization. However, these experiments have been carried out in the tunneling regime, where standard tunneling theory [19] predicts higher ionization rates for linear polarization. The results presented here are not strictly within the tunneling regime (due mainly to the relatively high frequency) and repre- sent new behavior. In Fig. 2(b), we have used the same parameters as in Fig. 1 (the spectra are rescaled such that the fundamental peak is equal to one in intensity for each ellipticity) and now find that the harmonics act as seen in experiments [9,10]: As the ellipticity is increased, the harmonics become less intense until no harmonics are discernible from noise. Only the first four harmonics can be seen for $\epsilon=0.6$, and the peaks are found to be rather broad, indicating that they are generated on a short time scale. In addition, there seems to be a correlation between the ionization behavior and the ellipticity beyond which the harmonics are destroyed: The normalization flattens out after approximately $\epsilon=0.7$, which is also the ellipticity at which the harmonics disappear.

To highlight the very different behavior of the ionization for various ellipticities, we have calculated the normalization at the end of the pulse for linear and circular polarization as a function of intensity (Fig. 3). At low intensities we see the expected perturbative multiphoton behavior, observed in experiment [10], which is that linear polarization produces a greater amount of ionization because of the selection rule restrictions on circular polarization transitions. However, as the intensity is increased to the critical intensity for over the barrier ionization (about 0.005 a.u. of intensity), we observe that the behavior changes such that circular polarization becomes more efficient in producing ionization; and, indeed, for much of the intensity range shown it is at least an order of magnitude greater. Note that the normalization curve for linear polarization is not smooth: This was previously observed by Pindzola and Dörr [20] and LaGattuta [21] and assigned to ac Stark shifted resonances and threshold effects. However, we find, as in Ref. [21], that for circular polarization these structures do not occur, perhaps because of the more restrictive circular dipole selection rules, at least in the perturbative regime of intensities.

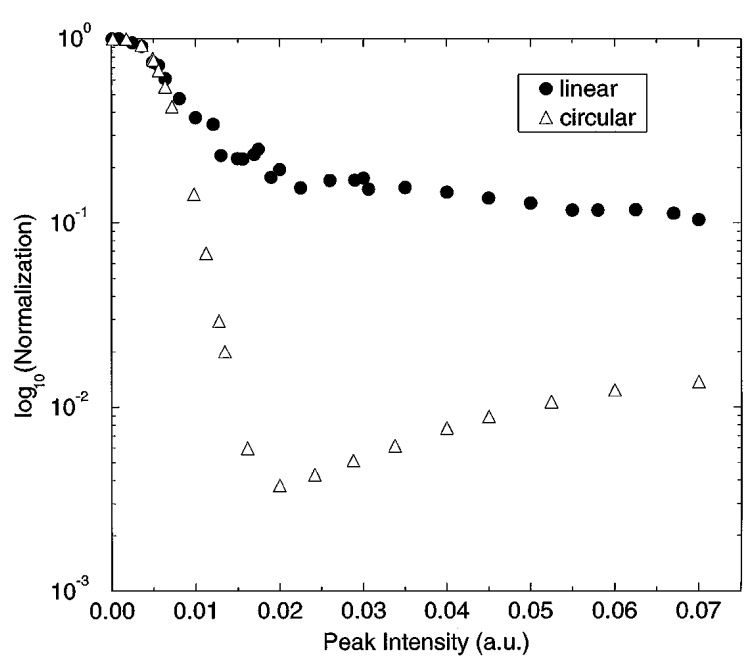

FIG. 3. Normalization as a function of peak laser intensity for a six-cycle pulse with wavelength $526 \mathrm{~nm}$, for linear and circular polarization. 
Finally, we observe a minimum in the normalization implying that stabilization is occurring. This is in agreement with calculations made by Pont and Gavrila [22] for circular polarization using time-independent high frequency Floquet theory. The lowest frequency which they have used is $\omega=0.125$ a.u., while we have found stabilization, albeit not to the same degree, for a frequency 1.5 times smaller. Interestingly, for the same parameters, linear polarization shows no signs of stabilization. This may be due to the atom not surviving the passage through "death valley" [23]. We are currently investigating adiabatic stabilization with much higher frequencies and intensities, the results of which will be presented elsewhere.

In summary, we have investigated numerically, with two-dimensional $a b$ initio calculations, the dependence of ionization and HHG on ellipticity. In particular, we have found that the harmonics are destroyed by elliptical light, as seen in recent experiments $[9,10]$. In a parameter regime where the tunneling picture is not entirely valid, circular polarization produces a greater amount of ionization than linear. We have also found evidence for stabilization in circularly polarized light, in qualitative agreement with previous time-independent calculations. The method we propose opens up the possibility of studying fully quantum-mechanically wave-packet evolution in time-dependent ellipticities proposed for the generation of ultrashort pulses of harmonics [24]. As well as this exciting prospect, the unified description of the strong-field phenomena, based on the exact wave-packet dynamics presented here, will open the way to resolve problems associated with, e.g., the accuracy of the "two-step" model for harmonic generation, time-dependent laser polarization, control of harmonic generation in time, ionization with elliptical light, elliptical stabilization of wave packets, angular distribution of photoelectrons in ATI, and many other important questions in strong-field physics that could be addressed only by approximate theoretical models in the past.

We would like to thank J.H. Eberly and D. D. Meyerhofer for useful discussions. This work has been funded in part by the UK Engineering and Physical Sciences Research Council and the European Union. One of us (D. G. L.) acknowledges support through the EU Training and Mobility of Researchers Scheme.
[1] For a recent review, see, e.g., M. Protopapas et al., Rep. Prog. Phys. 60, 389 (1997).

[2] J. H. Eberly et al., in Atoms in Intense Laser Fields, edited by M. Gavrila (Academic, New York, 1992), p. 301.

[3] K. C. Kulander et al., in Atoms in Intense Laser Fields, edited by M. Gavrila (Academic, New York, 1992), p. 247.

[4] R. M. Potvliege and R. Shakeshaft, in Atoms in Intense Laser Fields, edited by M. Gavrila (Academic, New York, 1992), p. 373.

[5] X. Tang et al., Phys. Rev. Lett. 65, 3269 (1990); E. Cormier and P. Lambropoulos, J. Phys. B 30, 77 (1997).

[6] M. Dörr et al., Phys. Rev. Lett. 71, 3943 (1993).

[7] E. Huens et al., Phys. Rev. A 55, 2132 (1997).

[8] Huens et al. [7] have been able to circumvent, to some degree, these problems by using Coulomb-Sturmian basis functions, but they are able to study cases with high frequency only.

[9] K. Budil et al., Phys. Rev. A 48, R3437 (1993); P. Dietrich et al., Phys. Rev. A 50, R3585 (1994); Y. Liang et al., J. Phys. B 27, 1296 (1994).

[10] Y. Liang, et al., J. Phys. B 28, 2757 (1995).

[11] R. Grobe and J.H. Eberly, Phys. Rev. A 48, 4664 (1993); D. G. Lappas et al., J. Phys. B 29, L619 (1996).

[12] M. D. Feit et al., J. Comp. Phys. 47, 412 (1982).

[13] K. C. Kulander et al., in Super-Intense Laser-Atom Physics, edited by B. Piraux, A. L'Huillier, and K. Rzążewski, NAO Advanced Study Institute, Series B: Physics Vol. 316 (Plenum Press, New York, 1993), p. 95.

[14] P. B. Corkum, Phys. Rev. Lett. 71, 1994 (1993).

[15] M. Lewenstein et al., Phys. Rev. A 49, 2117 (1994).

[16] M. Protopapas et al., Phys. Rev. A 53, R2933 (1996).

[17] T. Auguste et al., J. Phys. B 25, 4181 (1992).

[18] S. Augst and D.D. Meyerhofer, Laser Phys. 4, 1155 (1994).

[19] M. V. Ammosov et al., Zh. Éksp. Teor. Fiz. 91, 2008 (1986) [Sov. Phys. JETP 64, 1191 (1986)].

[20] M. S. Pindzola and M. Dörr, Phys. Rev. A 43, 439 (1991).

[21] K. J. LaGattuta, Phys. Rev. A 43, 5157 (1991).

[22] M. Pont and M. Gavrila, Phys. Rev. Lett. 65, 2362 (1990).

[23] M. Gavrila, in Atoms in Intense Laser Fields, edited by M. Gavrila (Academic, New York, 1992), p. 435.

[24] P. B. Corkum et al., Opt. Lett. 19, 1870 (1994); M. Yu. Ivanov et al., Phys. Rev. Lett. 74, 2933 (1995); C. Altucci, C.-G. Wahlström, and A. L'Huillier (private communication). 